\title{
The CFD Simulation for the West Pipeline Engineering on the Contamination Levels of Crude \& Product Oil
}

\author{
Liang Yue ${ }^{1, a}$, Xin Yanping ${ }^{1, b}$ \\ ${ }^{1}$ College of Oil and Gas Engineering, Shengli College China University of Petroleum, Shandong \\ Dongying, China \\ aliangyue87@163.com, '934694569@qq.com
}

Keywords: West Oil Product Pipeline; Crude Oil; Oil Product; Contaminated Concentration; CFD simulation

Abstract. West Oil Product pipeline always take batch transportation, conveying three different qualities of oil product. Crude oil pipeline takes a task of oil transportation from three oilfields in Xinjiang. In accordance with the principles of higher prices for better quality of crude oil, it takes batch transportation finally. The paper takes a $10 \mathrm{~m}$ three-dimensional vertical pipe as a carrier. It takes two kinds of oil alternative transportation upward or downward. When the transport order, gravity, temperature are different, in order to reduce the volume of the mixing oil and optimize the batch transportation program, we have the concentration of follow-up oil product \& the variation of follow-up oil product in different section after Simulation.

West trunk Pipeline is from Urumqi to Terminal Station of Lanzhou pass by Xinjiang and Gansu provinces about a total of 21 cities. It is a major project relating to people's livelihood and its real length is about $1858 \mathrm{~km}^{[1]}$. Beginning of the design is crude oil blending transportation, because the data about physical testing and modification for crude oil did not completely obtained. There was difference of crude oil physical properties in three oilfields in Xinjiang, it takes batch transportation finally. West Oil Product pipeline always take batch transportation. In order to make the optimal solution for the batch transportation, alternating hot and cold crude oil transport modeling in Different delivery conditions, and alternating product oil transport modeling between two qualities quite different oil products. Provide valuable reference for the company's operations and management of pipelines and facilities after Simulation.

\section{The Physical Model for Batch Transportation of Pipeline in One Ditch}

Based on the prototype of West Pipeline (Crude Pipeline $\varphi 813 \mathrm{~mm} \times 11 \mathrm{~mm}$; Products Pipeline $\varphi 559 \mathrm{~mm} \times 7 \mathrm{~mm}$ ), the length of model is $10 \mathrm{~m}$. Grid Generation of Calculation Region is hexahedral mesh, which is shown in Fig.1.

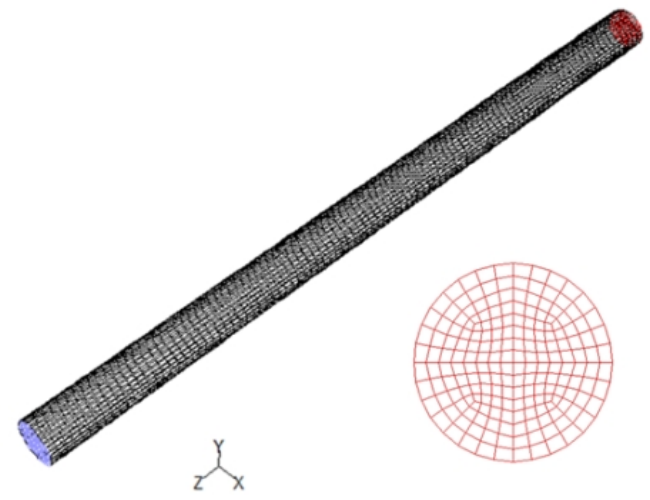

Fig.1 The three-dimensional-grid model of pipe ( $\&$ the grid of section)

According to the diffusion of composition and conservation equation of convection $\&$ reaction source, through an iterative process of FLUENT. Material mixing and transport processes can be simulated without chemical reactions. This paper is the simulation of alternate delivery of different substances, So we can use no chemical reaction component transport model ${ }^{[2]}$. Several oil physical parameters are shown in Table. 1 in the contamination of oil. 


\begin{tabular}{lllll}
\hline & $\begin{array}{l}\text { Density } \\
(\mathrm{kg} / \mathrm{m} 3)\end{array}$ & $\begin{array}{l}\text { Dynamic Viscosity } \\
(\mathrm{mPa} \cdot \mathrm{s})\end{array}$ & $\begin{array}{l}\text { Thermal Conductivity } \\
\mathrm{W} /\left(\mathrm{m} \cdot{ }^{\circ} \mathrm{C}\right)\end{array}$ & $\begin{array}{l}\text { Specific Heat } \\
\text { Capacity } \mathrm{J} /\left(\mathrm{kg} \cdot{ }^{\circ} \mathrm{C}\right)\end{array}$ \\
\hline Gasoline90\# & 738 & 0.546 & 0.16 & 2040 \\
No.0 diesel & 847.4 & 2.966 & 0.16 & 1905 \\
Tuha oil $\left(20^{\circ} \mathrm{C}\right)$ & 815.3 & 5.55 & 0.17 & 1760 \\
Beijiang oil $\left(40^{\circ} \mathrm{C}\right)$ & 838.69 & 14.8 & 0.16 & 1820 \\
Beijiang oil $\left(50^{\circ} \mathrm{C}\right)$ & 831.96 & 9.99 & 0.16 & 1860 \\
\hline
\end{tabular}

Table.1 Physical Properties of the oil for intermixing simulation

The Simulation for the West Pipeline Engineering on the Contamination condition of Crude \&

\section{Product Oil}

2.1 The effects of transport order and gravity differences on contamination in pipeline

A case study of batch transportation take with two petroleum products (Gasoline90\# and No.0 diesel ) in West oil product pipeline. The diameter and of tube is $559 \mathrm{~mm} \times 7 \mathrm{~mm}$, velocity is $1.276 \mathrm{~m} / \mathrm{s}$, the length of erect pipeline is $10 \mathrm{~m}$. When upward transport oil, the concentration distribution of contamination of oil product and the variation of follow-up oil product in different section are shown in Fig.2.
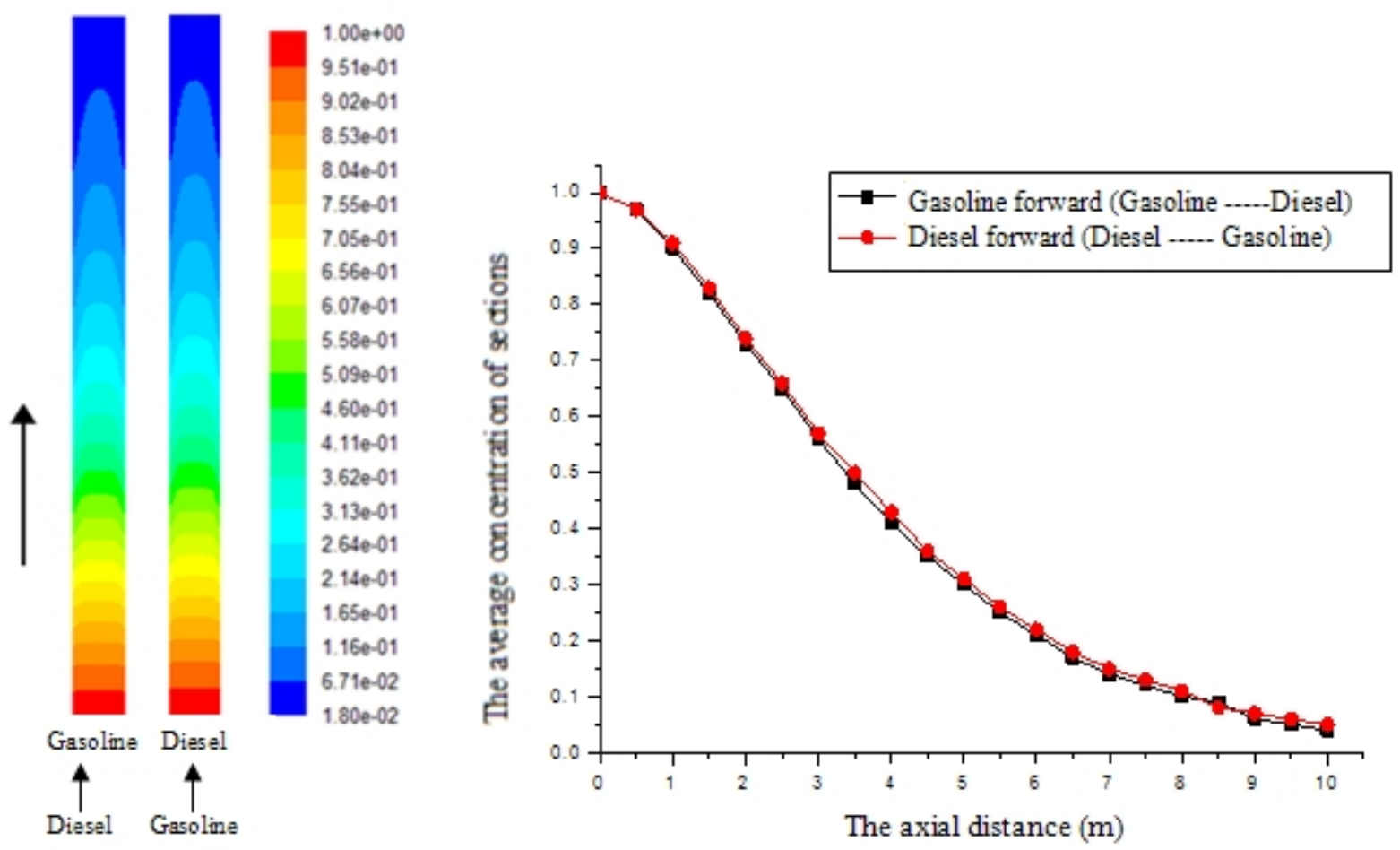

Fig. 2 The concentration nephogram of follow-up 0il \& The variation of follow-up 0il Product in different section(Upward transport oil)

The Fig. 2 show that when the transmission sequence is Gasoline 90\# forward and No.0 diesel follow-up, the concentration distribution in different section is uniform. Because turbulence intensity depending on the material distribution in mixing section, which is different from homogeneous area. In the process of No.0 diesel forward, mass force and density of diesel are large. But the speed of laminar sublayer is very small, and less inhibited in mass force. There is more mixed oil quantity in diesel forward than gasoline forward ${ }^{[3]}$.

The interface length formed by No.0 diesel forward and Gasoline90\# follow-up is longer than Gasoline90\# forward and No.0 diesel follow-up, and mixed oil tail with diesel concentration smaller is formed. Because batch transportation take with two petroleum products, oil follow-up wedge to oil forward, pipe wall would have Liquid mold of oil forward. With the occurrence of the oil flow, liquid mold will flow. In the same case of the transport, laminar boundary layer of oil with viscosity larger is 
thicker. Its viscous force will enhance, which led mixed oil quantity of oil forward adherent wall becomes much, follow-up of low-viscosity oil is difficult to wash away the viscous flow boundary layer of oil left in the low turbulent mixing intensity near the wall. In addition, diesel forward with larger density and turbulence intensity of laminar sublayer is small, oil suffered a more significant impact in mass force. So weakening the gasoline follow-up to the wall of diesel forward of erosion.
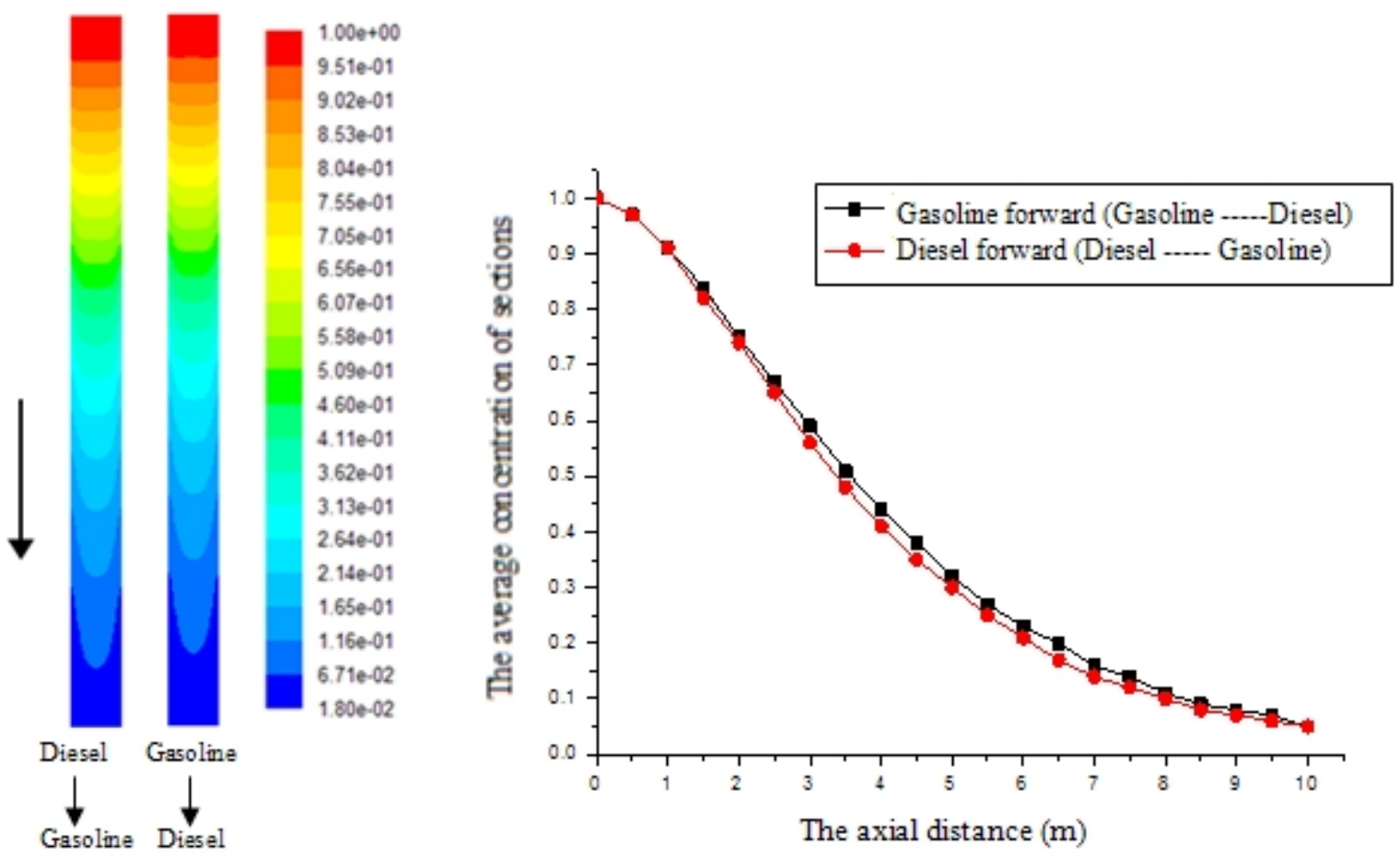

Fig.3 The concentration nephogram of follow-up Oi Product \& The variation of follow-up Oil Product in different section(Downward transport oil)

When downward transport oil, the concentration distribution of contamination of oil product and the variation of follow-up oil product in different section are shown in Fig.3. If oil with high density follow-up, high density of oil will make subsequent gravitational potential energy increases, Which flows into the front line of gasoline. Contaminated interface ultimately towards the bottom pipe, situation shown in Figure 3. There is more mixed oil quantity in Gasoline forward than diesel forward.

It shows that we should follow a logical sequence of petrol and diesel for transport. Compared with gasoline and diesel in the uphill process, mixed oil quantity and interface length of the former is relatively large. Compared with gasoline and diesel in the downhill process, mixed oil quantity and interface length of the former is relatively small. Therefore it should not be believed that any of the high viscosity oil forward's interface length longer than low viscosity oil forward.

2.2 The Simulation for the Contamination condition of cold and hot Crude Oil batch transportation

A case study of batch transportation take with two petroleum products (tuha oil and beijiang oil ) in West crude oil pipeline. Exploration key indicators for the contaminated concentration of batch transportation concentration have an effect contaminated influences. Because there is a large dissimilarity about rheology and physical characteristics, tuha oil is high pour point and low viscosity oil, and beijiang oil is high pour point and high viscosity oil. Both are related to heating delivery problems, so two petroleum under different transport temperature were numerically simulated ${ }^{[4]}$. Crude pipeline $\varphi 813 \mathrm{~mm} \times 11 \mathrm{~mm}$, the length of model is $10 \mathrm{~m}$. When upward transport oil, the contaminated concentration distribution of Crude oil, and the variation of follow-up crude oil in different section are shown in Fig.4.

As shown as Fig.4, when the transmission sequence is tuha oil forward and beijiang oil follow-up, the concentration distribution in different section is Uniform, whose density and viscosity 
are small. When the contaminated zone temperature changes and the oil flow, the density and viscosity of tuha oil forward are smaller, the adhesion of the inner wall is reduced. The Fig. 4 show that mixed oil tail of tuha oil forward is longer, due to liquid film formed by tuha oil flowing through the pipe wall thinner than beijiang oil, beijiang oil follow-up has high density and viscosity. Easily washed away low viscosity oil remaining laminar boundary, the mixed oil tail of tuha oil forward is longer. When the transmission sequence is beijiang oil forward and tuha oil follow-up, due to liquid film formed by beijiang oil flowing through the pipe wall thicker than tuha oil and turbulent kinetic energy near the wall lower, so mixed oil tail is shorter. As the temperature of mixed oil increases, the density and viscosity of tuha oil follow-up are smaller, the adhesion of the inner wall is reduced, turbulent kinetic energy increase relatively. And more near the beijiang oil, the viscosity more lower and diffusion faster, so tuha oil has relatively high content of the mixed oil.
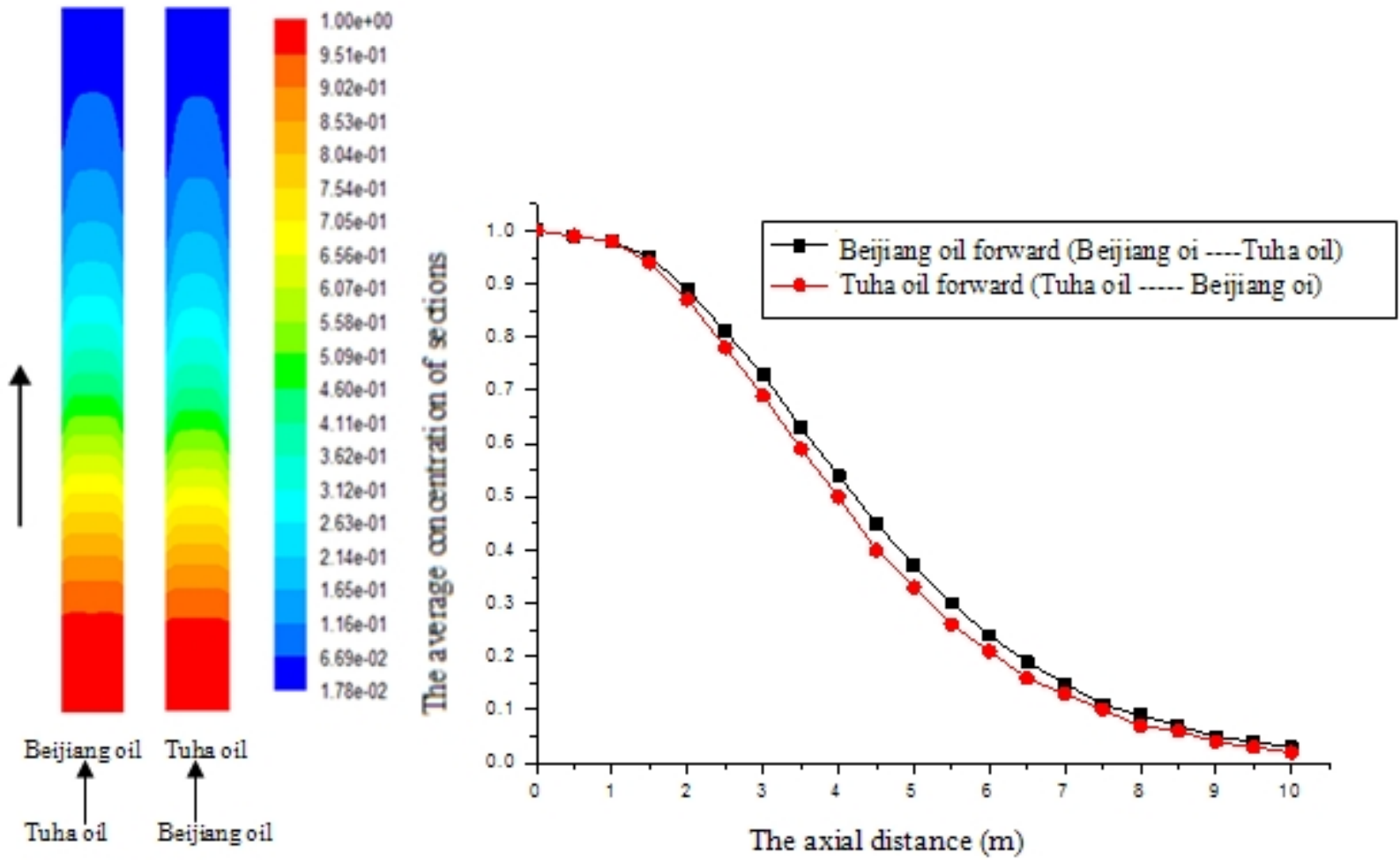

Fig.4 The concentration nephogram of follow-up crude oil \& The variation of follow-up crude oil in different section (beijiang oil in $50^{\circ} \mathrm{C}$, tuha oil in $20^{\circ} \mathrm{C}$,Upward ransport oil)

As shown as Fig.4, there is less mixed oil quantity in tuha forward than beijiang forward. Because the transmission sequence is beijiang oil forward and tuha oil follow-up, its density and viscosity are lager, and downward by mass force is lager. In contrast, the density and viscosity of tuha oil follow-up are smaller, the adhesion of the inner wall and mass force reduced. So it will continue to move forward wedged beijiang oil, resulting in an increase in the amount of contaminated.

Left of Fig. 5 is the concentration nephogram of beijiang oil follow-up in different temperature. When the other conditions remain unchanged, tuha oil forward has constant temperature, adjust the delivery temperature of beijiang oil follow-up, we found smaller temperature difference between the two petroleum products, mixed oil quantity more less. Because the viscosity and density of beijiang oil follow-up in $40^{\circ} \mathrm{C}$ higher than in $50^{\circ} \mathrm{C}$, making the downward force and the tube wall adhesion enhancement. So mixed oil quantity of beijiang oil in $40^{\circ} \mathrm{C}$ less than in $50^{\circ} \mathrm{C}$.

Right of Fig. 5 is the concentration nephogram of beijiang oil forward in different temperature. When the other conditions remain unchanged, tuha oil follow-up has constant temperature, adjust the delivery temperature of beijiang oil forward, we found the higher temperature of beijiang oil forward, the mixed oil quantity of tuha oil follow-up more less. Because the viscosity and density of beijiang oil reduced with the temperature higher, and making the downward force and the tube wall adhesion reduced. And the temperature of beijiang oil forward higher will lead contaminated zone temperature 
rise. Resulting in the viscosity and density of tuha oil follow-up began to decline, and making the downward force and the tube wall adhesion reduced, further increasing the amount of oil wedging in the beijiang oil.
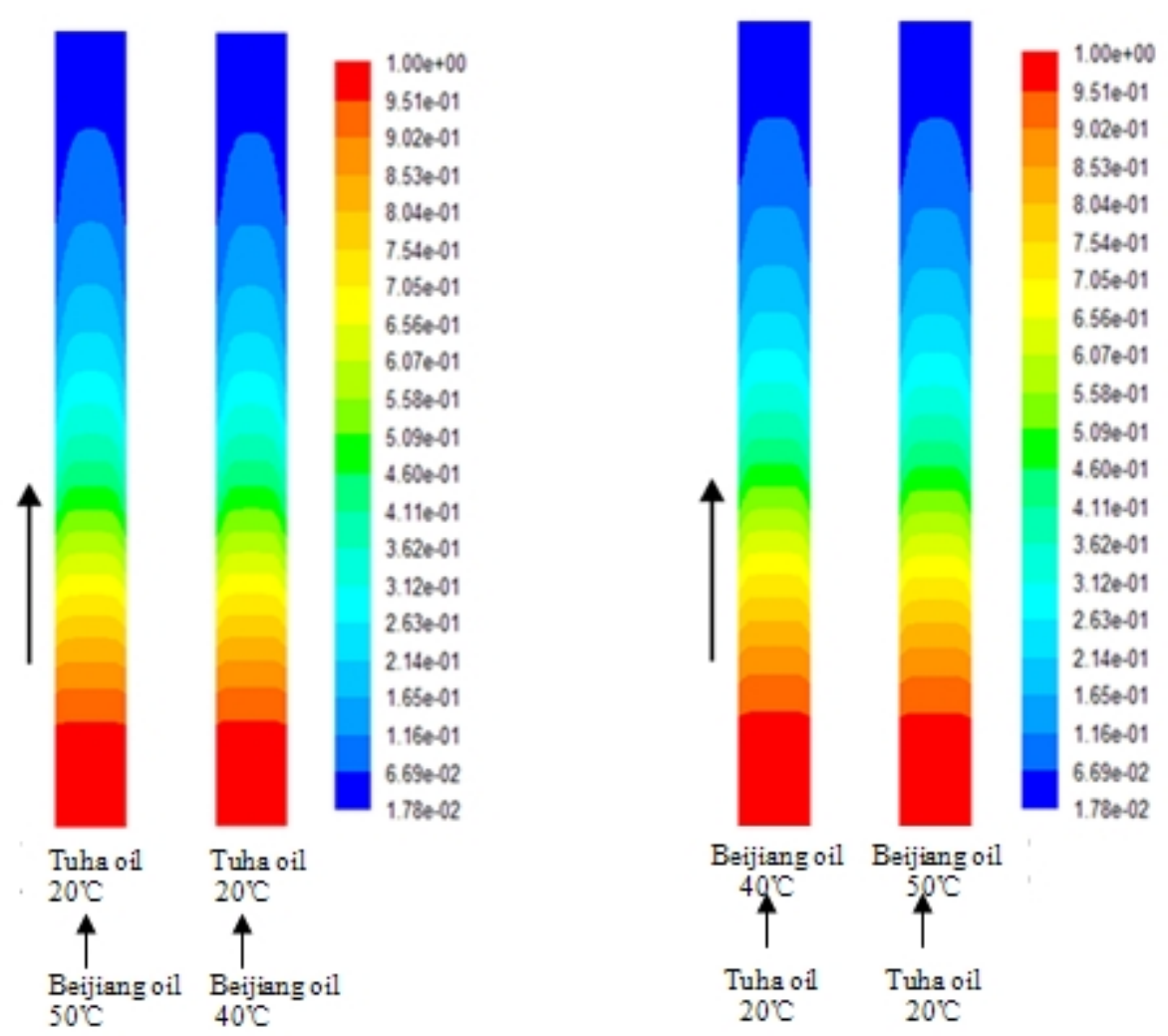

Fig.5 The concentration nephogram of follow-up crude oil (Upward ransport oil)

\section{Conclusions}

When the products Pipeline take batch transportation, mixed oil quantity can be reduced by gasoline forward and diesel follow-up in the uphill process, and mixed oil quantity can be reduced by diesel forward and gasoline follow-up in the downhill process.

When the crude Pipeline take batch transportation, at a flow rate unchanged, the pattern of tuha forward and beijiang follow-up can significantly reduce the amount of crude oil contaminated. And smaller temperature difference between the two petroleum products, mixed oil quantity will fewer. When the transportation is and tuha follow-up, the temperature of beijiang forward higher, mixed oil quantity will fewer.

\section{References}

[1] West Crude \& product Oil Pipeline Project General Information, B. File No.Chu-6000/Ming,2005:18

[2] Li Pengfei, Xu Minyi, Wang Feifei, Proficient in CFD engineering simulation and actual cases, People Post Press, Beijing, 2011,pp.55-60.

[3] Pu Jiangning, Problems of the Contamination of Batching Transportation of Products, J. OGST. 2000, 19(2)21-24.

[4] Cui Xiuguo, Zhang Jinjun, The research of heat transfer problem in process of batch transportation of cool and hot oil, J. OGST,2004,23(11)15 19 
